\title{
Erratum to: Analysis of epidermal lipids in normal and atopic dogs, before and after administration of an oral omega-6/ omega-3 fatty acid feed supplement. A pilot study
}

Iuliana Popa • Didier Pin • Noëlle Remoué • Bilal Osta • Sylvie Callejon • Emilie Videmont • Hugues Gatto • Jacques Portoukalian • Marek Haftek

Published online: 11 November 2011

(C) Springer Science+Business Media B.V. 2011

Erratum to: Vet Res Commun (2011) 35:8 501-509

DOI 10.1007/s11259-011-9493-7

The original version of this article unfortunately contained an error in the authors' names. The first name of the third author should be Noëlle instead of Nathalie.

The authors' names should read: Iuliana Popa · Didier Pin · Noëlle Remoué · Bilal Osta · Sylvie Callejon · Emilie Videmont · Hugues Gatto · Jacques Portoukalian · Marek Haftek

The online version of the original article can be found at http://dx.doi. org/10.1007/s11259-011-9493-7.

I. Popa $\cdot$ N. Remoué • B. Osta $\cdot$ S. Callejon · J. Portoukalian •

M. Haftek

Université Lyon 1, Laboratory for Dermatological Research EA4169

"Normal and Pathological Functions of the Skin Barrier",

Lyon, France

D. Pin $\cdot$ E. Videmont

Dermatology-Cancerology Unit, VetAgro Sup Lyon Campus,

Marcy L'Etoile, France

H. Gatto

Virbac SA,

Carros, France

M. Haftek $(\bowtie)$

Laboratoire de Recherche Dermatologique, EA4169,

Université Lyon 1,

8, Av. Rockefeller,

69373 Lyon, France

e-mail: marek.haftek@univ-lyon1.fr

Present Address:

I. Popa

UMR CNRS 8612, University of Paris XI,

92290 Chätenay-Malabry, France 